\title{
Mothers and Pediatricians: Self-Power Versus Knowledge
}

\author{
Simone De Carvalho* and Jose Martins Filho \\ Universidade de Campinas - Saúde da Criança e do Adolescente, Brazil
}

*Corresponding author: Simone De Carvalho, MEd, PhD Candidate, Universidade de Campinas - Saúde da Criança e do Adolescente, Brazil

\begin{abstract}
Objective: To analyze the perception of the pediatric guidance by the mothers attended in private offices, aiming to know how they assimilate, process and use the information received by their pediatrician.

Method: Data collection was realized through a questionnaire sent to participants via e-mail and recorded the interview. A total of 200 mothers from a virtual community in the social network participated in the research. The answers were transcribed using the Discourse of the Collective Subject method. The analysis was supported by the qualitative research perspective, from the Social Representation Theory.

Results: The following categories were obtained through data analysis: (1) Being prepared to discuss pediatrician conducts; (2) Conquering mastery of their maternal specificities; (3) Empowerment and its contribution to mother-pediatrician dynamics. These categories demonstrate that the mothers' level of knowledge of pediatric subjects and their ability to use them in decision-making about the care of their babies showed a direct relation between whether or not to follow the pediatrician's guidance.

Conclusion: The mothers' opinions shows that the action on the decision to keep up with the health of their baby is directly proportional to the quality of the pediatric care, such as: (1) The certification of the recommendations updated and proven in agreement with the official health care guidelines and; (2) Support and recognition by the pediatrician of self-power acquired by the mother. The practice of access to knowledge of these mothers regarding the care of their baby difficulties the pediatric follow-up. This is due to the distrust in the confront between the information previously acquired by the mother and the pediatric guidance.
\end{abstract}

\section{Keywords}

Pediatrician, Child guidance, Women, Maternal and child health

\section{Introduction}

The mother's comprehension of her baby's care is influenced by affective sociocognitive factors. Studying the impact of maternal emotional availability, Fonseca and Silva [1] observes that the quality of the support of health professionals in the follow-up and orientation of the mother - in which the mother is most affectively and materially supported by a social environment she will have a better understanding of her baby. The importance of maternal empowerment as a behavior that gives mothers self-control over their baby's health decisions is the goal of this study.

For Martins Filho [2], the mother's sadness, unrest and fear in caring for and breastfeeding her baby is not always understood by the family, especially the partner, who, in general, is not used to this feeling never presented in other periods of her life. This sense of weakness is mainly reflected in situations in which hospitals and maternity units do not have staff and a team sensitized, where the pediatrician's support role becomes essential.

Cherry and Gibson [3] point out that frustration with the health care system is experienced when mothers realize that their usual habits of care haven't been considered effective, in their point of view, by health professionals. Mothers advocate for their children because they understand that they are individuals who depend on them for the care and need to speak on their behalf, once they are unable to do so - this is because mothers respond emotionally, cognitively, and behaviorally to the received orientations. In emotional responses, mothers emotionally involved with their baby usually take responsibility for their health and their baby. 
In the report of the mothers of the study, many found in the hierarchical environment of the doctor's office the difficulty of fully exercising the sense of self-power over the life of their child, in which medical protocol became primordial in care. The authors state that "only mothers can discover the unique characteristics of their child through care" [3]. Mothers wanted, actually, the acknowledgment and respect of their opinions about their children's health and experienced a real sensation of being understood by wother mothers. The authors conclude that mothers are the driving force to constantly move the perceptions of health professionals because they know in depth what is best for their children very often.

Mothers in this context seek to define and redefine their roles in a constant understanding of the specific care of their baby by building their autonomy. This process, especially in virtual groups, has been increasingly evidenced, through the mothers' decisionmaking, as they are more and more aware of the knowledge about child development, getting deeper and seeking scientific knowledge, and are continually questioning the professional practice, which is often not consistent with the confrontation of these acquired knowledge [4].

For Andrade [5], the pediatrician needs to be culturally sensitive to understand who this mother is, her social and family context, besides realizing what's her idea of motherhood, for through this prior knowledge, it would be possible to achieve adequate care based on empathy. The mutual sharing of knowledge, experience and acquirements between the pediatrician and the mother has the potential to provide satisfactory followup and evaluation as long as the communication is effective.

The scientific relevance of this study about the maternal perception of pediatric care is established at the interface of both the importance of the recognition of maternal care as a fundamental factor in the quality of the child's health in the medium and short term, as well as in the expansion of the evaluation of the pediatrician in the maternal universe and how he or she can take a more in-depth orientation based on the mother's unique perceptions of her baby. Therefore, its development and continuity is necessary, aiming at a broader and more sophisticated action of prevention in children's health and the quality of the exercise of childcare in pediatric care.

\section{Methodology}

The present study is the result of qualitative and quantitative research of a descriptive exploratory type that aimed to interpret the discourses of mothers who participate in a virtual motherhood support group regarding their perception of pediatric care and the consequent decisions about their baby's health.
The virtual community analyzed in the social network Facebook has been working for five years as a support group that daily act is guiding and sharing knowledge and experiences with thousands of mothers through the internet. The goal of this community is the maternal self-empowerment in the network. These mothers, after being guided by the moderators of the groups, support and guide each other. The richness and variety of this virtual sharing network allow us to understand the dynamics of this relationship and its practical implications in the lives of mothers and, consequently, their babies, by the aims of this research.

The main reason that leads mothers to seek the virtual community, in general, is the experience of some difficulty in the postpartum, especially with the moment of breastfeeding. The community keeps up with this mother in the daily dynamics of the involvement and care of her baby. Usually, the mother participates in the virtual groups according to her needs and remains a member of the groups, following the daily publications and actively participating in the life of the community, through discussions and sharing of her personal experiences regarding her maternal processes.

For the composition of the data of this study, different data collection strategies were used, incorporating qualitative and quantitative research techniques, investigating their interfaces, with the aim of seizing more broadly the opinions of the mothers participating in the study. The choice of this qualitative method suggests a fundamentally humanized approach, in which the comprehensive study of the discourses looks promising to comprehend the real meaning of the experiences lived by these subjects. It satisfies the need for on approach that contemplates the existential totality of the researched subjects, their daily lives, cultures, and personal experiences. The rigor of its applicability, attests the solidity of its results since it isn't the results of the systematic procedures adopted by the researcher, but the variables that are revealed during the study, in such a way that both the participant and the reader can identify yourself in the results obtained [6].

The quantitative data of the research were collected in the Statistical Package for the Social Sciences (SPSS 18.1) for the descriptive analysis of the universe of the surveyed mothers through inferential statistics - a method that makes it possible to estimate the characteristics of a population from samples, with qualitative and quantitative variables. The quantitative data are presented descriptively in Tables/Graphs by using the SPSS statistical data processing package. The data collected through the form were statistically analyzed, as well as the variables for the population sample, through a descriptive analysis of the data obtained. The exploratory analysis of data was also performed, through summary measures (frequency, percentage, mean, standard deviation, minimum, median and maximum). The 
association of the variables was tested using the exact Fischer or Chi-square test (categorical), Kruskal-Wallis or Mann-Whitney (numerical). The adopted level of significance was $5 \%$. The first part of the questionnaire encompassed the basic information and maternal history and the second part, the history of pediatric care.

Qualitative research finds more, and more space in the health area and its reliability is recognized by its capacity to reflect the thinking of the individuals researched, in their essence and nature. Minayo [7] states that "as in any social project, the 'health' object offers a possible level of quantification but surpasses it when it comes to understanding deep and meaningful dimensions that cannot be imprisoned in variables" [7]. The perspectives of the researcher or the social observer are facilitated by a deep involvement with the research universe, as an integrant of the process being investigated, making it able to interpret the phenomena more accurately and attribute more meanings to the researched realities.

Qualitative data were organized and analyzed through the program Discourse of the Collective Subject (DSC), software QualiQuantiSoft for the construction of the single subject of social representation. The DSC is a Brazilian method of tabulation and organization of qualitative data that is based on the Theory of Social Representation [8]. Social representations (SR) are sociocognitive schemes that allow associating thoughts, beliefs, and values of these representations at the objective characteristics of the speech owners. The choice and use of this method as a research tool occurred so that it was possible to know the thoughts and representations of this researched universe of mothers. This method allows the exposure of collective thinking in a single subject, in which she/he will speak for all the mothers interviewed. It is the self-expression of collective thought in itself, which cannot be quantified [9].

Created and developed by Lefèvre [10], the Discourse of the Collective Subject (DSC) is elaborated with parts of speeches with the same meaning by standardized means of the program, thus adding the statements of the mothers without reducing them, but incorporating them into the collective discourse, always in the first person singular. In it, it is possible to gather distinct discourses or thoughts of a group of individuals, under unique categories. It is the study of art, extracting the essence of these mothers' discourses that may be a way of understanding the dialogue between the pediatrician and the mother. The process of analyzing the data collected in the study occurred in two moments: (1) Central ideas (unifying category) and; (2) Anchorages or key expressions (discourse of the collective subject) that make up each testimony included in the research. The central ideas $(\mathrm{Cl})$ describe, in a synthetic way, the meanings present in the analysis of the individual responses of the mothers, while the expressions (EC) are important passages of the discourses that deserve to be highlighted in full, according to the adopted theory. Its organization takes place through the composition and the literal transcriptions of each collected response, which constitute the essence of the representations content, evidenced in the stories of the participants. This organization can describe, in a synthetic but precise way, the sense present in the EC, where each response, by way of example, allows one or more central ideas to be found.

Social representations are clear arguments of the processes of social changes and their behaviors. Through SR, it is possible for individuals to construct theories about their social objects and to communicate, in a clear and comprehensible way to their reality, the diverse organizations of behaviors they are inserted. This theory instigates an understanding of the diverse forms of thought and theories elaborated in daily life, seeking an explanation for the change of attitudes in the systems of social relationships. The SR theory investigates what a group of people think about a certain subject matter; how they interpret everyday events; how they form an opinion about them and how they behave in this reality.

Once one wishes to know, through empirical research, the opinions and thoughts of a given group of individuals, their beliefs, knowledge and collective experiences that interfere directly in the decision-making of their members and change of behavior, it becomes necessary the use of Social Representations Theory, an object of the present scientific investigation. We can say that in SR it does not make sense to say that its finding is a mistake because it is a form of a certain social thought whose specificity originates from the different situations of thought and the norms that shape it. Its qualitative basis highlights the opinions present in the conscience of the surveyed mothers about their vision of the health professional, the pediatrician.

The project was approved by the Research Ethics Committee of the Faculty of Medical Sciences of UNICAMP (CAAE: 33285414.0.0000.5404). All women participating in the study were approached as to their desire and consent to use the data written and recorded in the survey for the study. The entire procedure followed the requirements of resolution 466/2012 of the National Health Council (CNS/MS/BRAZIL).

\section{Results}

To understand the life history of the surveyed mothers, the place of the pediatric care was considered in the questionnaire to identify where these mothers are attended and the institution responsible for the baby's care. In the present study, the place of pediatric care is an important indicator of the type of care preferred by the mothers at the consult, since 185 mothers (92.5\%) opted for the private health system while 14 mothers (7.5\%) opted for the public health system. Such preference for 
private pediatric care was due in part to the choice of mothers for the follow-up of the baby's health offered by the professional who was (or appeared to be) more in line with their personal preferences and values. Of the 200 (100\%) mothers participating in this study, the majority were in the age group of 31 to 40 years, in a total of 120 participants, corresponding to $60 \%$ of the interviewees; followed by the group of women aged 20 to 30 years, in a total of 67 (33.5\%); and the smallest group, women aged 41 to 50 years, for a total of 10 (5\%). The average age of the mothers is 30 years. A total of 3 mothers did not report their age group (1.5\%). Of the 200 participants, 67 (33.5\%) are in the range of 6 to 10 minimum wages, which characterizes this segment as having a high middle class (C).

The second part of the questionnaire aimed to do a more detailed investigation of the experiences of mothers in the postpartum care along the pediatrician's follow-up. The objective was to identify the perceptions, wishes, and desires of these mothers in this first contact with this health professional. The last question, with descriptive qualitative character, had as objective to identify the degree of the mother's commitment to follow or not the pediatrician's guidance. Five research subcategories were created: 1) Initial doubts; 2) Orientation and support to breastfeeding; 3) Sensitive listening of the pediatrician in the postpartum period; 4) Use of language that is easy to understand and; 5) Prior knowledge about pediatric guidelines.

In the analysis of the quantitative data about having initial doubts answered by the pediatrician, $69.5 \%$ of the mothers answered yes, indicating that the first care was satisfactory for most of the surveyed mothers. In the evaluation of $51.5 \%$ of the mothers, however, the support and guidance received from the pediatrician were seen as negatively, demonstrating that the expectations about the performance of this health professional in the postpartum period, from the perspective of the mothers, are not satisfactory. About the action of sensitive listening by the pediatrician, especially related to their postpartum dilemmas, $55.0 \%$ of the mothers answered negatively, which leads us to conclude that both support and sensitive listening still leave gaps for the pediatrician's attendance to the mothers. We found out that $74.5 \%$ of the mothers answered positively about their doubts having been answered by the pediatrician, while $80.5 \%$ of them had previous knowledge about their doubts, evidence that the level of knowledge of the mother is a facilitator in understanding the pediatric orientation [11].

The qualitative exploratory interview with a deepening of the research theme, through the Skype online resource for free voice calls, is an easily accessible tool for reaching a population in several places, in real time. From the 40 mothers interviewed individually, the following Brazilian states are represented: São Paulo
(21), Paraná (6), Rio Grande do Sul (5), Rio de Janeiro (2), Santa Catarina (1), Mato Grosso do Sul (1), Goiás (1) and Pernambuco (1). The interviews were conducted from the one guiding question, scheduled by the mothers.

The ease of access to this virtual resource was to provide the mother a comfortable interview and according to her needs - the maternity leaves period, as an example. Not being mandatory the physical presence of the interviewee for the research, the virtual tool has practically unlimited reach. Another feature is the recording application for Skype, which automatically records all calls without time limit, creating a digital file immediately after the connection ends on the researcher's computer, allowing the transcription of the recordings as well as consult the file, if necessary. The video feature was not activated in the search, respecting the privacy of the mothers' image, according to the TCLE.

Thus, it was possible interview with ample possibilities of investigation and access of the mothers, who were able to explore the proposed theme and freely verbalize their ideas, feelings, and impressions about it, in an environment of freedom and comfort. The transcripts of the 40 interviews were included in the DSC program and analyzed according to the software steps, systematically. Some interviews had more than one Central Idea, and several tabs were created corresponding to the inclusion of the description of each mother, to compose the table of all the associated $\mathrm{Cls}$ and then analyzed and categorized by the similar discourse of the collectivity.

The acquisition of the knowledge by the surveyed mothers brings us the sense of the appropriation of the access to shared knowledge and experiences in support groups, as a way of constant evaluation of pediatrician behaviors and orientations, and the awareness of an egalitarian part on dynamics of child care. The dialogue becomes a facilitator of the pediatric guidance and the idea that the mother is an assiduous and integral observer of her baby and appropriates the updated knowledge in these interactions, finding her role of importance in this relationship at the time of the pediatric consultation. It is the appropriation of knowledge as a facilitator in pediatric relationships in the maternal view:

"I know a lot more about my baby than the pediatrician does. I know my baby. The pediatrician is a generalist... way what applies to one situation may not apply to another. I decide what is best for my child" (DSC 2).

From this thematic category, the following subcategories have emerged:

\section{Being prepared to discuss the behaviors of the pe- diatrician}

"The mother is the best person to tell what is happening... The pediatrician is not a king. If I sense some- 
thing is wrong, then I can't have an open relationship with him/her anymore" (DSC 2).

The mothers intend in their formulated impressions, through the diverse opinions and shares about the pediatric conducts of which they had access, to give proper meaning in their care of the baby, making this a possible action to be modified and improved based the exchange of experiences with your pediatrician. The mother's unique character of specific knowledge about her son contributes to an individualist discourse on the part of the pediatrician, both about the treatment and the follow-up of the baby, who can understand its specificities through the maternal impressions.

"It is hard to find a pediatrician you can trust... Regardless of the pediatrician, we always have to do our research... I bring my knowledge, and the pediatrician brings his. I bring my knowledge as a mother, out of my daily life, and he brings his pediatric knowledge... we put everything together and then each person goes his/her own way. $70 \%$ of my empowerment came through exchanging information with other mothers. I don't know whether I would have breastfed for so long if I had not talked with other mothers and opened my mind to certain things" (DSC2).

Self-power with respect to trust in the apprehension of her personal maternal experience and the need of an openness dialogue between the pediatrician's scientific knowledge, demonstrate an idea of the need to practice the mother's self-confidence about her personal experiences. The questioning action about the orientations received is the result of the direct relationship with their knowledge and self-power acquired.

\section{Conquering mastery of their maternal specificities}

"Of course, the pediatrician is a health professional; he studied for it, and he knows much more than I do. But I am not dumb: I can see and analyze things... I read a lot, mostly information from the World Health Organization. So, the pediatrician and I talk 'like equals'. I realize he is very careful with the mothers who have the knowledge and are empowered. Sometimes the pediatrician has good intentions... If the mother is not empowered, the doctor does what is easiest for him because a mother who is not empowered accepts whatever the pediatrician says" (DSC2).

Self-power is evident in the mothers' confidence in their ideas, in their perceptions of motherhood, and that has real meaning for them. The social representation of this action reveals the understanding of various forms of the thoughts of these women, reflecting the desires attributed to the meanings and constructing the social reality and the materialization of the ideas and intentions on this maternal dynamic.

"Pediatricians treat empowered mothers differently. So, my pediatrician always listens to me with respect be- cause he knows I do my research... He is careful about everything he says to me... Things have improved after I started to inform myself. This has made the pediatrician's work lighter in fact" (DSC2).

We can consider the attribution by mothers of their scientific knowledge through research and read about the subject, as an action of interference in pediatric practice. Since the pediatrician disregards the fact that mothers search other sources for validation of their orientation, the pediatrician's behavior depends on the mother's acceptance or not of following his guidance.

\section{Empowerment and its contribution to the mother- pediatrician dynamics}

"And I have already explained to my friends that it depends a lot of the attitude towards the doctor. I've always been very honest about everything I think. So, if he suggests something to me that I disagree with, I speak up. I no longer have that fear that I once had to face the doctor, to argue with him just because he is a doctor. We have, I think, the view that the doctor knows more because he is a doctor, and, in fact, he may have studied whatever he did, but he is human too, they can wrong too, right? And, of course, if the pediatrician is flexible, we can reach a middle ground for each one, I think, we end up knowing the limit what can we arrive .... And it's good, I think it is cool when you have that freedom with the doctor (...). Our relationship is an exchange. He knows that I question, that I ask, that I suggest hypotheses and we respect each other. Even though he knows my way, he respects me and it's very cool. He never treated me arrogantly even when I didn't agree with what he was talking about (...)" (DSC2).

The shared action of knowledge among mothers and their applicability in the decisions about their baby's care interpret the pediatrician no longer as the holder of all knowledge but a posture of mutual agreement between the role of the pediatrician and the maternal role. The mother, and her acquisition of knowledge and awareness of the role of the pediatrician, conquer through her empowerment a dialogical relationship and constant partnership. The exchange of ideas, opinions, hypotheses and personal opinions about the maternal practice and the recognition of them by the pediatrician, result in the satisfaction of the pediatric care.

"I am a health worker, and I know that some doctors think they are 'demigods'... Things have to change because we have this 'informed' dialogue, otherwise the relationship will become increasingly more difficult" (DSC2).

For the mothers of this study, the future of a pleasant relationship on their part of the pediatric service that meets their expectations regarding the follow-up of their baby is based on the practice of constant exchanges between both. It is fundamental for a facilitating action beyond the systematic accompaniment of the 
baby, contributing to the integral vision of the pediatrician of maternal and child health.

"The pediatrician must become a "friend", you know - not intimate - more like a person with whom you can have an open dialogue. She is a human being, not just a "mommy". She is a mother, a wife, a professional: she knows what her conditions are, what she intends to go with her motherhood... I think this is the future of Pediatrics and even of mothering as a whole - a dialogue between mothers, pediatricians, and society" (DSC2).

The practice of the empowerment dynamics can reach not only the mother and her baby but also the social context in which they are inserted. The condescension of the mother figure in her universe of conquered singularities and qualities, corroborates the pediatrician's recognition that this mother changed, became more demanding and have a certain amount of knowledge; and wishes her maternity to be recognized not as an isolated action of the baby's care, but with meaning for her own life, full of meanings.

"I do not think we're in the world to be passive. I think we need more "empowered" pediatricians and not pediatricians who only medicalize... who treat all babies the

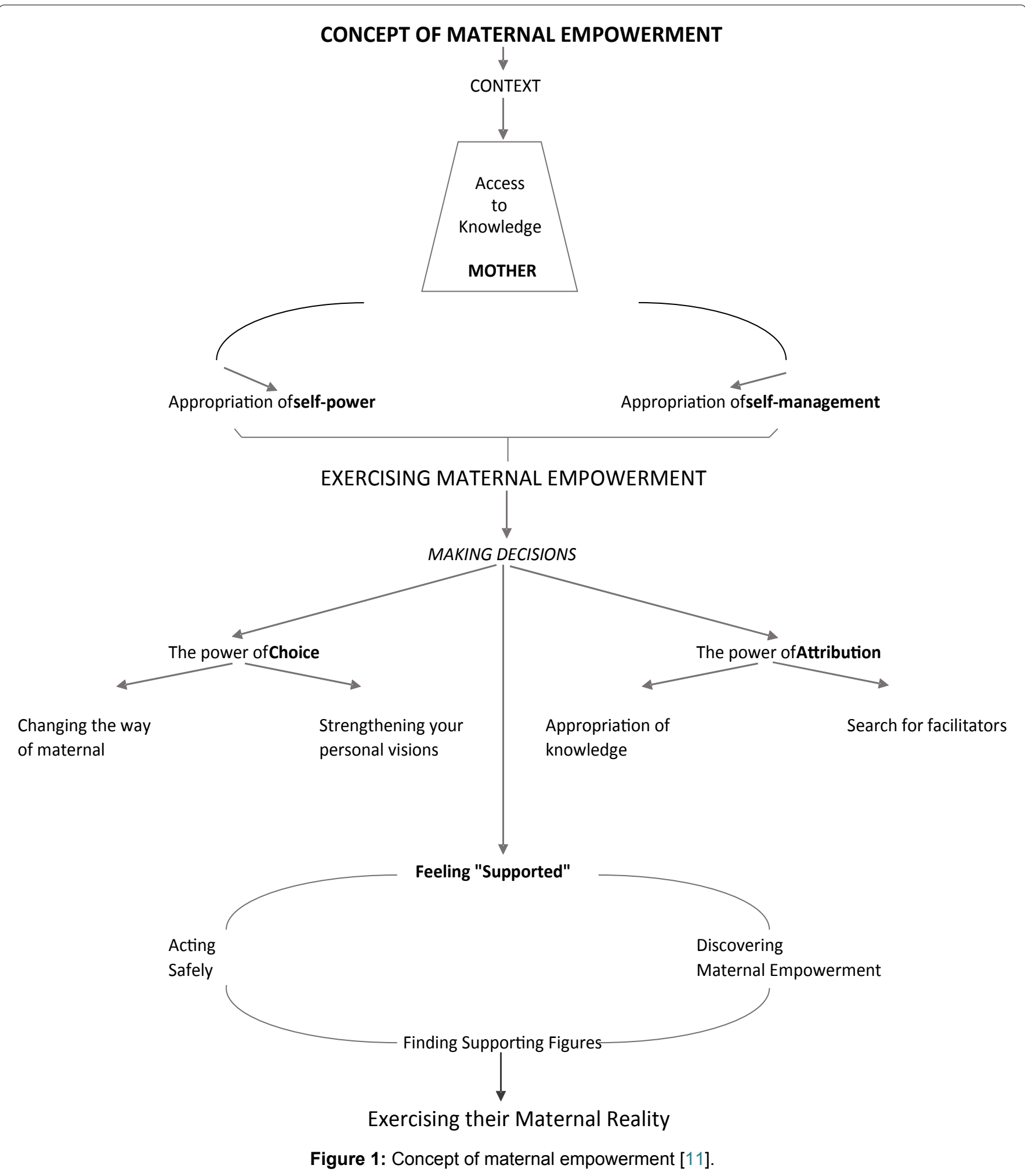


same. I want pediatricians who show the mother the entire development of the child, who can be trusted, who says that crying is normal, that breastfeeding is normal, that sometimes it is hard for the child to start eating and that the mother does not need to despair about it... That's what we need: 'empowering pediatricians to better help the mothers" (DSC2).

The attributed meaning of the idea of "empowering pediatricians" is the level of understanding of these mothers about the importance of redefining the roles of this professional, able to effectively contribute to their self-management in the care of their baby, based in integral support. Mothers who receive this kind of support from the pediatrician prove that interpersonal processes are increasingly necessary for the health area and the progress of the acceptance of maternal empowerment as a facilitating agent in the pediatrician's action (Figure 1).

\section{Discussion}

Women from different Brazilian states were heard, resulting in an abundant, and diversified material of different maternal ideas and experiences, mainly on the pediatrician's action in different regional contexts. The study aimed to describe and explain the behavior of these women, most of whom are middle class, with purchasing power and higher education, exploring their social environment and identifying their specificities as mothers, as well as their decisions regarding pediatric care received, his visions about his work of this health professional, as well as the propagation of this modality of communication through the maternal support virtual group in question.

The research made evident that the virtual world provides virtual spaces of interaction, in which mothers can openly express their opinions, knowledge, practices and emotions related to the experience of motherhood. Increasingly, mothers turn to the Internet in search of these shared network actions, facilitated by the growing use of computers and cell phones that allow them access to this world. The so-called 'social networks' have also become virtual spaces that promote, on a large scale, counseling and support actions with the creation of free discussion groups on the most varied topics of interest to its participants [12].

There is a recurrent behavior evidenced in most of the mothers' speeches about their constant reflection on the pediatric guidance received and the knowledge of the recommendations of the national and international health agencies through free access to this information on the Internet or discussed in the virtual groups mentioned. Mothers constantly share what several pediatricians have advised them on a particular subject, share their doubts to the group, share texts and acquired knowledge about the issues that interest them, they chat the information acquired and - usually at the next visit - discuss with the pediatrician what they have discovered, opting in some cases to change their pediatrician and even to abandon the care offered by this health professional [13].

The mothers researched in this context, share the information provided by the pediatrician to ensure that the guidance received are consistent with the practice of their maternal and solid support group. The decision whether or not to follow the pediatrician's orientations is strongly related to what each mother believes to be correct from her empowerment. If the mother identifies similarities in the ideas shared by other mothers, she tends to put into practice the actions that seem coherent and fair to her, which may influence her decision to follow her pediatrician's directions or not. The mother's safety in her actions and decisions is strengthened by the pediatrician's sensitivity to her ideas and practices, understood by her as being consistent with her personal experiences as a mother and an active participant in her maternal reference group [14].

The pediatrician's position about the affirmation of the scientific evidence-based guidelines that the mothers acquire through this knowledge also contributes to the confrontation of the familiar influences on the care of the baby [15]. Mothers seek to understand their maternal processes by approaching their own knowledge, often using words and senses from their repertoire of the environment, as well as the impressions that pass through the support groups, manifesting through them judgments and positions.

\section{Final Considerations}

The diversity of concrete opinions of the mothers involved in this process, based on the subjectivity of the collective discourse, reveal the dynamics between what the pediatrician orients and what the mother effectively does following the guidance. For the pediatrician, it is also important to consider the attendance of the mother; listening to her and guiding her in her initial doubts, insecurities, and mainly, being a support figure regarding her decisions and peculiar intentions to the exercise of her motherhood. The mother is an assiduous and fundamental observer as a figure of support for the quality of care of the pediatrician. The pediatrician is only heard and followed in this context when (1) He recognizes the mother's capacity for self-management, (2) Is up to date on with the specialization, and (3) Develops an interaction with this mother who is both sensitive and appreciates the maternal protagonism in the care of her baby.

Consequently, the mother's decision to follow the guidelines of the health professional is dependent on the quality of pediatric care in the following areas: certification of up-to-date recommendations and proven in agreement with official health agencies, and the discovery of support and recognition by the pediatrician 
of the mother's acquired power. The higher the level of this knowledge, the greater the need for a service based on the sharing of knowledge, recognition of the perceptions acquired by the mothers and their safety in exercising their maternal empowerment.

\section{References}

1. Fonseca VR, Silva GA, Otta E (2010) The relationship between postpartum depression and maternal emotional availability. Cad Saude Publica 26: 738-746.

2. Martins Filho J (2014) Birth and the family: Joys, surprises and concerns. Campinas: Papirus.

3. Gibson $\mathrm{CH}$ (1995) The process of empowerment in mothers of chronically ill Children. J Adv Nurs 21: 1201-1210.

4. Moraes IM (2010) Vulnerability of the patient versus individual autonomy. Rev Bras Saúde Mater Infant 10: S331-S336.

5. Andrade GRB, Vaitsman J (2002) Social support and networks: Connecting solidarity and health. Science \& Collective Health 7: S925-S934.

6. Merighi MAP, Square NS (2003) Qualitative theoreticalmethodological approaches: The experience of women in the reproductive period. Rio de Janeiro: Guanabara Koogan.

7. Minayo MCS (2000) The challenge of knowledge: qualitative research in health. São Paulo - Rio de Janeiro: Ed. Hucitec - Abrasco.
8. Moscovic S (1978) The Social Representation of Psychoanalysis. Rio de Janeiro: Zahar.

9. Figueiredo MZ, Chiari BM, Goulart BNG (2013) Discourse of the Collective Subject: A brief introduction to the qualitative research tool. Disturbed Comum 25: S129-S136.

10. Lefevre F, Lefevre AMC, Marques MCC (2009) Discourse of the collective subject, complexity and self-organization. Science and Collective Health 14: 1193-1204.

11. Carvalho S, Martins Filho J (2016) Family relationship with pediatricians: The maternal views. Rev Paul Pediatr 34: 330-335.

12. Gallegos D, Bennet-Russell R, Previte J, Parkinson J (2014) Can a text message a week improve breastfeeding? BMC Pregnancy Childbirth 14: 374.

13. Broom A (2005) Virtually healthy: The impact of internet use on disease experience and the doctor-patient relationship. Qual Health Res 15: 325-345.

14. Armstrong N, Powell J (2009) Patient perspectives on health advice posted on Internet discussion boards: A qualitative study. Health Expect 12: 313-320.

15. Bartlett YK, Coulson NS (2011) In the investigation into the empowerment effects of using online support groups and how this affects health professional/patient communication. Patient Educ Couns 83: 113-119. 